\title{
Regional and global antimicrobial susceptibility among isolates of Streptococcus pneumoniae and Haemophilus influenzae collected as part of the Tigecycline Evaluation and Surveillance Trial (T.E.S.T.) from 2009 to 2012 and comparison with previous years of T.E.S.T. (2004-2008)
}

Viktorija Tomic $^{1 *}$ and Michael J Dowzicky ${ }^{2}$

\begin{abstract}
Background: We report here on 14438 Streptococcus pneumoniae and 14770 Haemophilus influenzae isolates collected from 560 centres globally between 2004 and 2012 as a part of the Tigecycline Evaluation and Surveillance Trial (T.E.S.T.).

Methods: MIC testing was performed using broth microdilution methods as described by the Clinical and Laboratory Standards Institute (CLSI) using CLSI-approved breakpoints; US Food and Drug Administration breakpoints were used for tigecycline as CLSI breakpoints are not available.

Results: At least $99 \%$ of S. pneumoniae isolates globally were susceptible to levofloxacin, linezolid, tigecycline or vancomycin. Penicillin resistance was observed among $14.8 \%$ of S. pneumoniae and was highest in Asia/Pacific Rim (30.1\%) and Africa (27.6\%); $23.4 \%$ of S. pneumoniae isolates were penicillin-intermediate, which were most common in Africa (37.6\%). Minocycline susceptibility among S. pneumoniae decreased by 20\% between 2004-2008 and 2009-2012. High (>98.5\%) susceptibility was reported among $H$. influenzae to all antimicrobial agents on the T.E.S.T. panel excluding ampicillin, to which only $78.3 \%$ were susceptible. $\beta$-lactamase production was observed among $20.2 \%$ of $\mathrm{H}$. influenzae isolates; $1.5 \%$ of isolates were $\beta$-lactamase negative, ampicillin-resistant.
\end{abstract}

Conclusions: S. pneumoniae remained highly susceptible to levofloxacin, linezolid, tigecycline and vancomycin while H. influenzae was susceptible to most antimicrobial agents in the testing panel (excluding ampicillin).

Keywords: Antimicrobial susceptibility, Streptococcus pneumoniae, Haemophilus influenzae, Tigecycline

\section{Background}

Streptococcus pneumoniae and Haemophilus influenzae are among the most common causes of pneumonia worldwide [1,2]. According to the most recent guidelines for the treatment of community-acquired pneumonia (CAP) published by the Infectious Diseases Society of America and

\footnotetext{
* Correspondence: viktorija.tomic@klinika-golnik.si

'University Clinic of Respiratory and Allergic Diseases, Golnik 36, 4204 Golnik, Slovenia

Full list of author information is available at the end of the article
}

American Thoracic Society [3], the recommended empiric therapy for inpatient CAP includes a $\beta$-lactam (cefotaxime, ceftriaxone or ampicillin-sulbactam) plus either a respiratory fluoroquinolone or azithromycin for intensive care unit (ICU) patients or a respiratory fluoroquinolone or $\beta$-lactam plus a macrolide for non-ICU patients. European guidelines are also available [4], while several European countries have established their own policies regarding the treatment of CAP including the UK [5], Sweden [6] and the Netherlands [7]. These guidelines 
often differ in their treatment recommendations, even when bacteriology is similar between countries [8].

The Tigecycline Evaluation and Surveillance Trial (T.E.S.T.) is a global surveillance study which has been ongoing since 2004. This study was designed to monitor the longitudinal activity of a broad panel of comparator agents, including the glycylcycline antimicrobial tigecycline, against a collection of clinically important pathogens. Tigecycline possesses potent in vitro activity against those pathogens most often responsible for community-acquired pneumonia [9]. In 2009, the US Food and Drug Administration approved tigecycline for the treatment of community-acquired bacterial pneumonia (CABP) caused by Streptococcus pneumoniae (penicillin-susceptible isolates), including cases with concurrent bacteremia, Haemophilus influenzae ( $\beta$-lactamase negative isolates), and Legionella pneumophila in the USA [10], based in part on results from a pair of Phase III clinical trials comparing the efficacy of tigecycline and levofloxacin in hospitalized patients with CABP [11]. In the current study, we examine susceptibility trends among isolates of S. pneumoniae and H. influenzae collected from Africa, the Asia/Pacific Rim, Europe, Latin America, the Middle East and North America between 2004 and 2012.

This report updates $S$. pneumoniae and $H$. influenzae data previously reported from T.E.S.T. between 2004 and 2008 [12]. In that report, a total of 6785 S. pneumoniae and $6642 \mathrm{H}$. influenzae were analyzed. Penicillin resistance among $S$. pneumoniae ranged from $9.3 \%$ in Europe to $25.1 \%$ in the Asia-Pacific Rim and $\beta$-lactamase producing H. influenzae ranged from $8.7 \%$ in South Africa to $26.8 \%$ in the Asia-Pacific Rim. The $\mathrm{MIC}_{90} \mathrm{~s}$ for tigecycline against $S$. pneumoniae and $H$. influenzae were $\leq 0.12 \mathrm{mg} / \mathrm{L}$ and $\leq 2 \mathrm{mg} / \mathrm{L}$, respectively. Data from that 2004-2008 report are included in the dataset used in this report. This report aims to provide an update to Darabi et al. [12], comparing 2004-2008 data with data collected between 2009 and 2012.

\section{Methods}

Isolate collection

Between 2004 and 2012, a total of 560 centres globally contributed isolates to the T.E.S.T. study in at least a single study year (Table 1). All centres participating in T.E.S.T. were required to contribute a minimum of 65 Gram-positive and 135 Gram-negative isolates per study year; these were to include at least 15 isolates of both S. pneumoniae and $H$. influenzae annually. North America and Europe were the main contributors, with 221 and 196 centres participating in the two regions, respectively.

All body sites were considered acceptable sources for isolate collection; a maximum of $25 \%$ of isolates in any year could be urinary in origin. Banked or stored isolates were not accepted, nor were duplicate isolates from a single patient. Isolates were collected from 13 different body sites (Table 2); a small number of isolates were collected from medical instruments. Isolates were included into the study if they were considered a clinically relevant causative organism and the probable causative agent of the infection. Patient age, medical history or gender were not considered relevant. Isolate identification was carried out by contributing centres using routine local methodologies.

The central repository for all isolates was International Health Management Associates (IHMA, Schaumburg, IL, USA), who were responsible for organism transport and collection, confirmation of isolate identification and the management of a database including all isolate data. Quality control testing was carried out daily using $S$. pneumoniae ATCC 49619, H. influenzae ATCC 49247 and $H$. influenzae ATCC 49766. Approximately 10-15\% of isolates were randomly tested annually by IHMA to verify isolate identity and MICs; MIC data were used only if daily QC results were within acceptable ranges as published by the Clinical and Laboratory Standards Institute (CLSI) [13].

Table 1 Number of centres contributing S. pneumoniae or H. influenzae isolates between 2004 and 2012

\begin{tabular}{lllllllllll}
\hline Region $^{\mathbf{a}}$ & $\mathbf{2 0 0 4}$ & $\mathbf{2 0 0 5}$ & $\mathbf{2 0 0 6}$ & $\mathbf{2 0 0 7}$ & $\mathbf{2 0 0 8}$ & $\mathbf{2 0 0 9}$ & $\mathbf{2 0 1 0}$ & $\mathbf{2 0 1 1}$ & $\mathbf{2 0 1 2}$ & Total $^{\mathbf{b}}$ \\
\hline Africa & 1 & 4 & 4 & 6 & 2 & 2 & 0 & 1 & 8 \\
Asia/Pacific Rim & 7 & 6 & 20 & 24 & 17 & 14 & 12 & 1 & 13 & 48 \\
Europe & 31 & 25 & 50 & 70 & 104 & 112 & 95 & 35 & 108 & 196 \\
Latin America & 4 & 13 & 22 & 24 & 35 & 29 & 15 & 6 & 25 & 59 \\
Middle East & 1 & 2 & 3 & 5 & 9 & 9 & 9 & 4 & 7 & 20 \\
North America & 70 & 110 & 99 & 92 & 45 & 43 & 32 & 23 & 80 & 221 \\
Global & 114 & 160 & 198 & 221 & 212 & 209 & 163 & 70 & 241 & 560 \\
\hline
\end{tabular}

${ }^{\mathrm{a} A f r i c a}=$ Mauritius, Morocco, South Africa, Tunisia; Asia/Pacific Rim = Australia, China, Hong Kong, India, Malaysia, Pakistan, Philippines, Singapore, South Korea, Taiwan, Thailand; Europe = Austria, Belgium, Bulgaria, Croatia, Czech Republic, Denmark, Finland, France, Germany, Greece, Hungary, Ireland, Italy, Latvia, Lithuania, Norway, Poland, Portugal, Romania, Slovak Republic, Slovenia, Spain, Sweden, Switzerland, The Netherlands, United Kingdom; Latin America = Argentina, Brazil, Chile, Colombia, Guatemala, Honduras, Jamaica, Mexico, Panama, Venezuela; Middle East = Israel, Jordan, Kuwait, Lebanon, Oman, Saudi Arabia, Turkey; North America $=$ Canada, United States.

${ }^{\mathrm{b}}$ Total $=$ the total number of unique centres contributing isolates between 2004 and 2012. 
Table 2 Geographic distribution and culture source $(>5 \%$ of total) of $S$. pneumoniae and $H$. influenzae isolates

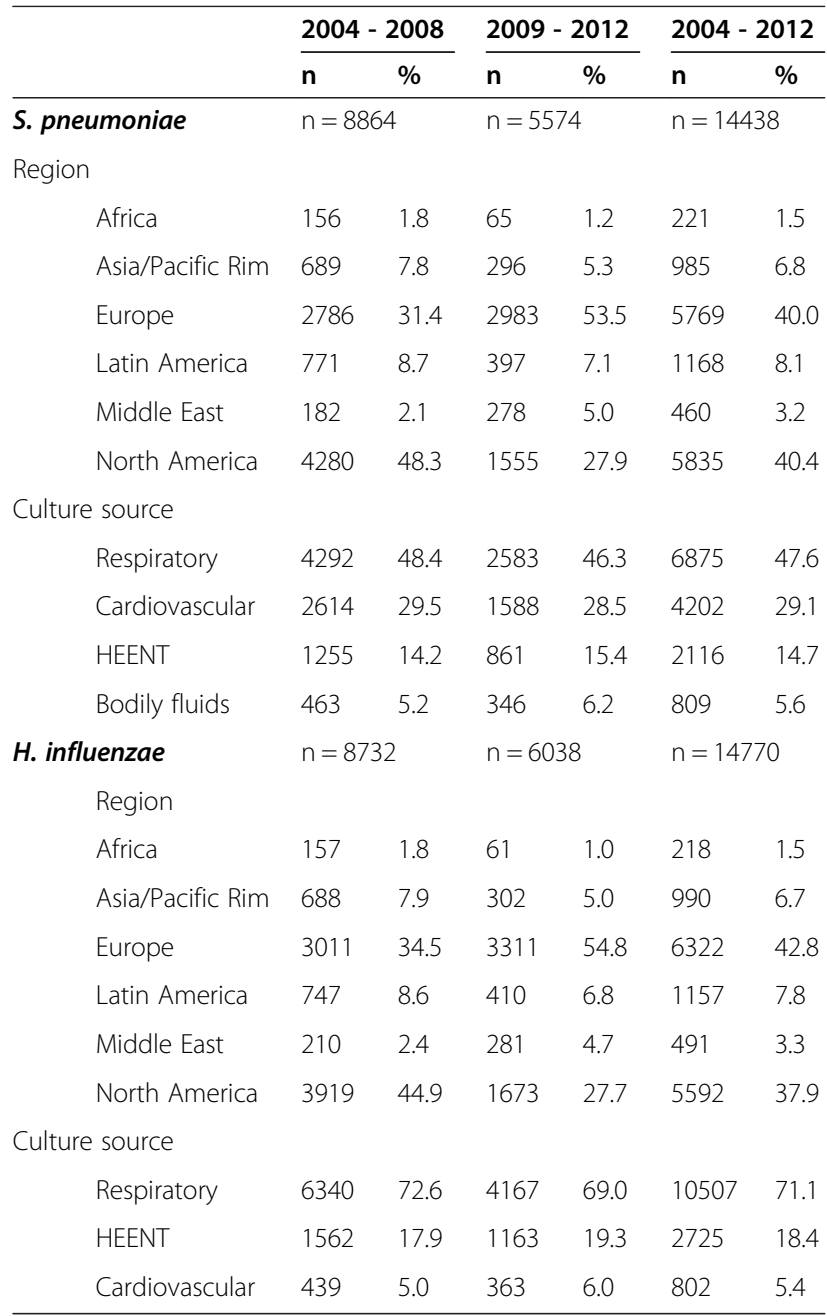

Bodily fluids includes abscess/pus, abdominal, bile, cerebrospinal fluid, pericardial, peritoneal, pleural, synovial and tissue; Cardiovascular includes cardiovascular, blood, blood vessels and heart; HEENT includes head, ears, eyes, nose and throat; Respiratory includes respiratory, bronchial brushing, bronchials, bronchoalveolar lavage, endotracheal aspirate, lungs, sinuses, sputum and trachea.

\section{Antimicrobial susceptibility testing}

All centres were responsible for minimum inhibitory concentration (MIC) testing using broth microdilution methods described by CLSI [14] using either MicroScan ${ }^{\circledR}$ panels (Dade Microscan Inc., West Sacramento, CA, USA) or Sensititre ${ }^{\bullet}$ plates (TREK Diagnostic Systems, East Grinstead, UK). The T.E.S.T. test panel for S. pneumoniae included the following antimicrobial agents: amoxicillinclavulanate, ampicillin, ceftriaxone, imipenem, levofloxacin, linezolid, meropenem, minocycline, penicillin, piperacillintazobactam, tigecycline and vancomycin. Azithromycin, clarithromycin, clindamycin and erythromycin were added to the T.E.S.T. panel for S. pneumoniae in 2008, with older isolates tested retrospectively. $H$. influenzae MICs were determined using a panel which included amikacin, amoxicillin-clavulanate, ampicillin, cefepime, ceftriaxone, imipenem, levofloxacin, meropenem, minocycline, piperacillin-tazobactam and tigecycline. In 2006, imipenem testing with MicroScan ${ }^{\odot}$ plates was replaced in the T.E.S.T. panel with meropenem using Sensititre ${ }^{\oplus}$ plates due to imipenem stability problems.

Antimicrobial susceptibility was determined using CLSI-approved breakpoints [13] with the exception of tigecycline, for which US Food and Drug Administration (FDA)-approved breakpoints were used as CLSI breakpoints are not available for tigecycline [15]. Penicillin oral breakpoints (susceptible $\leq 0.06 \mathrm{mg} / \mathrm{L}$, resistant $\geq 2 \mathrm{mg} / \mathrm{L}$ ) were used for $S$. pneumoniae in this study; penicillinintermediate $S$. pneumoniae (PISP) was defined as a penicillin MIC between 0.12 and $1 \mathrm{mg} / \mathrm{L}$.

The MIC results for 2004-2008 presented here do not exactly match the results presented in Darabi et al. [12] due to retesting of selected isolates from 2004-2008 through the intervening study period or the deletion of untestable isolates from the T.E.S.T. database (most often due to loss of culture viability).

\section{Antimicrobial resistance determination}

$\beta$-lactamase production among $H$. influenzae was determined at collecting centres using preferred local methodologies. The central lab, IHMA, retested all $H$. influenzae if the ampicillin results did not agree with the $\beta$-lactamase determination. The central laboratory (IHMA) uses the cefinase test [15]. Only $15 \beta$-lactamase-negative, ampicillin-resistant (BLNAR) $H$. influenzae isolates were collected globally during this study; therefore, we have replicated the approach of Darabi et al. [12] here and have included $\beta$-lactamase-negative, ampicillinintermediate (BLNAI) isolates as well. This resulted in a total of 228 BLNAR isolates globally.

\section{Statistical analysis}

A comparison of rates of susceptibility between the two time periods 2004-2008 and 2009-2012 was carried out using the Fisher's Exact Test. The upper and lower 95\% confidence intervals (CI) presented in this manuscript relate to percentage susceptibility. The $95 \%$ CI for the percentage susceptible presented in this manuscript were calculated using the SAS exact method for binomial confidence intervals.

\section{Results}

\section{S. pneumoniae}

In total, $14438 \mathrm{~S}$. pneumoniae isolates were contributed to T.E.S.T. between 2004 and 2012 (Table 2). North America was the largest contributor of S. pneumoniae isolates between 2004 and 2008 (48.3\%) while Europe contributed the most isolates between 2009 and 2012 (53.5\%) (Table 2). The most common isolate sources for 
S. pneumoniae were respiratory ( $47.6 \%$ of all isolates) followed by cardiovascular (29.1\%) (Table 2).

Globally, S. pneumoniae was highly susceptible to vancomycin (100\%), linezolid (>99.9\%), tigecycline (99.9\%), and levofloxacin (>98.9\%) (Additional file 1: Table S1). The global susceptibility of $S$. pneumoniae to most other T.E.S.T. panel agents decreased slightly, often by $<1 \%$, between 2004-2008 and 2009-2012. The largest change was observed for minocycline: susceptibility to this agent decreased significantly $(\mathrm{p}<0.0001)$ from $72.1 \%$ in $2004-2008$ (95\% CI 71.2-73.0) to 51.7\% in 2009-2012 (95\% CI 50.453.0). This drop in minocycline susceptibility was observed in all regions, ranging from $15.5 \%$ in Europe to $31.7 \%$ in Latin America (Additional file 1: Table S1). Conversely, global susceptibility to meropenem and penicillin increased significantly $(\mathrm{p}<0.0001)$ between $2004-2008$ and 2009-2012; for meropenem from 79.5\% (95\% CI 78.480.5 ) to $83.4 \%$ (95\% CI 82.4-84.3) and for penicillin from $60.0 \%$ (95\% CI 59.0-61.1) to $64.8 \%$ (95\% CI 63.5-66.0). Meropenem susceptibility increased in all regions, although the change was only statistically significant in Europe $(\mathrm{p}<0.01)$ where susceptibility increased from $84.9 \%$ (95\% CI 83.3-86.5) in 2004-2008 to $88.0 \%$ (95\% CI 86.889.2) in 2009-2012. Penicillin susceptibility increased in Africa (11.7\%), Asia/Pacific Rim (3.5\%), Europe (0.9\%) and North America (6.0\%) but decreased in Latin America (7.2\%) and the Middle East (3.2\%). The change was only statistically significant in North America $(\mathrm{p}<0.0001)$ where susceptibility increased from 57.3\% (95\% CI 55.858.8 ) to $63.3 \%$ (95\% CI 60.9-65.7).

Some notable regional changes in S. pneumoniae susceptibility were reported which did not impact largely on global rates (Additional file 1: Table S1). In Asia/ Pacific Rim, decreases in susceptibility of between $8.3 \%$ and 16.0\% between 2004-2008 and 2009-2012 were seen for amoxicillin-clavulanate $(\mathrm{p}<0.001,95 \%$ CI 86.9-91.6; 76.2-85.4), ceftriaxone ( $\mathrm{p}<0.0001,95 \%$ CI 87.7-92.3; 72.982.6), azithromycin ( $\mathrm{p}<0.01,95 \%$ CI 44.6-53.0; 33.5-45.2), clarithromycin $(\mathrm{p}<0.01,95 \%$ CI 44.8-53.1; 33.5-45.2), erythromycin ( $\mathrm{p}<0.01,95 \%$ CI $44.5-52.8 ; 33.2-44.8)$ and clindamycin ( $\mathrm{p}<0.0001,95 \%$ CI 61.1-69.0; 43.2-55.1). In the Middle East, macrolide susceptibility decreased by approximately $10 \%$ although the decrease was not statistically significant.

Macrolide susceptibility was strongly influenced by penicillin non-susceptibility among $S$. pneumoniae. PISP isolates were $17 \%$ less susceptible to macrolides than all S. pneumoniae globally combined; susceptibility was reduced by approximately $45 \%$ among penicillinresistant S. pneumoniae (PRSP) isolates (Additional file 1: Table S1). Macrolide susceptibility among all S. pneumoniae varied widely by region in this study, ranging from $45 \%$ in Asia/Pacific Rim to $72 \%-73 \%$ in Latin America; macrolide susceptibility among PRSP ranged from $5 \%$ in Asia/Pacific Rim to $45 \%$ in Latin America.

Ceftriaxone susceptibility among S. pneumoniae isolates decreased in all regions between 2004-2008 and 20092012 , by $3.2 \%$ globally $(\mathrm{p}<0.0001)$ from $96.3 \%$ (95\% CI 95.9-96.7) to $93.1 \%$ (95\% CI 92.4-93.7) and by as much as $12.1 \%$ in the Asia/Pacific Rim [from 90.1\% susceptible (95\% CI 87.7-92.3) to 78.0\% (95\% CI 72.9-82.6)]. The decrease in ceftriaxone susceptibility was considerably more pronounced among PRSP isolates: susceptibility dropped by $\geq 18.8 \%$ in 5 of the 6 regions globally and by as much as $35 \%$ in Latin America, decreasing from $80.0 \%$ (95\% CI 71.1-87.2) to $45.0 \%$ (95\% CI 32.1-58.4) ( $<<0.0001)$, with a global decrease in susceptibility of $21.2 \%$ observed between $2004-2008$ (78.1\% susceptible, 95\% CI 75.8-80.3) and 2009-2012 (56.9\% susceptible, 95\% CI 53.4-60.4) $(\mathrm{p}<0.0001)$ (Additional file 1: Table S1).

Levofloxacin, linezolid, tigecycline and vancomycin retained similar activity against PRSP isolates compared with all S. pneumoniae isolates globally. All other antimicrobial agents showed decreases in activity against PRSP, as high as $45 \%$ among the macrolides and over 70\% among the carbapenems (meropenem and imipenem) (Additional file 1: Table S1). This was most noticeable in Asia/Pacific Rim, where PRSP susceptibility to the macrolides, ceftriaxone, clindamycin and minocycline (5\%, 56.4\%, $28.4 \%$ and $9.5 \%$, respectively) was much lower than global levels $(20-22 \%, 70.1 \%, 43.4 \%$ and 27.6\%).

Regarding resistance phenotypes, 3374 (23.4\%) were PISP while 2132 (14.8\%) were PRSP globally (Additional file 1: Table S1, Table 3). Regionally, PISP levels were highest in Africa (37.6\%), Latin America (31.3\%) and Middle East (30.7\%) during the complete study interval; PISP levels increased by almost 6\% in Latin America but decreased by $>18 \%$ in Africa, although these changes were not statistically significant. The prevalence of PISP decreased globally, from $25.0 \%$ (95\% CI 24.1-25.9) in 2004-2008 to $20.8 \%$ (95\% CI 19.7-21.9) in 2009-2012 $(\mathrm{p}<0.0001)$ (Table 3). PRSP isolates were most prevalent in Asia/Pacific Rim (30.1\%) and Africa (27.6\%) between 2004 and 2012; rates of PRSP increased in both of these regions between 2004-08 and 2009-12 ( $>4 \%$ and $>6 \%$, respectively) although the changes were not statistically significant. The prevalence of PRSP was stable globally, decreasing only slightly from $15.0 \%$ (95\% CI 14.2-15.7) in $2004-2008$ to $14.4 \%$ (95\% CI $13.5-15.4$ ) in $2009-2012$ (Table 3).

\section{H. influenzae}

A total of 14770 isolates of $H$. influenzae were contributed between 2004 and 2012 as a part of the T.E.S.T. study. North America was the largest contributor of H. influenzae isolates over 2004-2008 (44.9\%) while 
Table 3 Rates of PISP and PRSP plus $\beta$-lactamase-positive $H$. influenzae and BLNAR, regionally and globally

\begin{tabular}{|c|c|c|c|c|c|c|c|c|c|c|c|c|}
\hline & \multicolumn{3}{|l|}{ PISP n (\%) } & \multicolumn{3}{|l|}{ PRSP n (\%) } & \multicolumn{3}{|l|}{ BL-Pos HI n (\%) } & \multicolumn{3}{|l|}{ BLNAR $^{a}$ n (\%) } \\
\hline & $2004-08$ & 2009-12 & 2004-12 & $2004-08$ & $2009-12$ & 2004-12 & $2004-08$ & $2009-12$ & 2004-12 & $2004-08$ & $2009-12$ & 2004-12 \\
\hline Africa & $67 / 156(42.9)$ & 16/65 (24.6) & 83/221 (37.6) & 40/156 (25.6) & $21 / 65$ & $61 / 221(27.6)$ & 13/157 (8.3) & $6 / 61(9.8)$ & 19/218 (8.7) & $4 / 157(2.5)$ & $1 / 61(1.6)$ & $5 / 218(2.3)$ \\
\hline Asia/Pacific Rim & 152/689 (22.1) & 42/296 (14.2) & 194/985 (19.7) & 198/689 (28.7) & 98/296 (33.1) & 296/985 (30.1) & 192/688 (27.9) & $83 / 302(27.5)$ & $275 / 990(27.8)$ & $23 / 688(3.3)$ & $12 / 302(4.0)$ & $35 / 990(3.5)$ \\
\hline Europe & $536 / 2786(19.2)$ & $559 / 2983(18.7)$ & $1095 / 5769(19.0)$ & 295/2786 (10.6) & $302 / 2983(10.1)$ & $597 / 5769(10.3)$ & 436/3011 (14.5) & 499/3311 (15.1) & 935/6322 (14.8) & 56/3011 (1.9) & $45 / 3311(1.3)$ & 101/6322 (1.6) \\
\hline Latin America & 226/771 (29.3) & 139/397 (35.0) & $365 / 1168(31.3)$ & 105/771 (13.6) & 60/397 (15.1) & 165/1168 (14.1) & $154 / 747(20.6)$ & $84 / 410(20.5)$ & 238/1157 (20.6) & $8 / 747(1.1)$ & $12 / 410(2.9)$ & 20/1157 (1.7) \\
\hline Middle East & $54 / 182(29.7)$ & 87/278 (31.3) & $141 / 460(30.7)$ & 43/182 (23.6) & $70 / 278(25.2)$ & $113 / 460(24.6)$ & $42 / 210(20.0)$ & 53/281 (18.9) & 95/491 (19.3) & $1 / 210(0.5)$ & 4/281 (1.4) & $5 / 491(1.0)$ \\
\hline North America & $1180 / 4280(27.6)$ & $316 / 1555(20.3)$ & 1496/5835 (25.6) & $646 / 4280(15.1)$ & 254/1555 (16.3) & 900/5835 (15.4) & 1015/3919 (25.9) & 410/1673 (24.5) & 1425/5592 (25.5) & 40/3919 (1.0) & 22/1673 (1.3) & $62 / 5592(1.1)$ \\
\hline Global & 2215/8864 (25.0) & 1159/5574 (20.8) & $3374 / 14438(23.4)$ & 1327/8864 (15.0) & $805 / 5574(14.4)$ & 2132/14438 (14.8) & 1852/8732 (21.2) & 1135/6038 (18.8) & $2987 / 14770(20.2)$ & $132 / 8732(1.5)$ & 96/6038 (1.6) & $228 / 14770(1.5)$ \\
\hline
\end{tabular}

${ }^{a}$ BLNAR includes both $\beta$-lactamase negative, ampicillin-resistant and $\beta$-lactamase-negative, ampicillin-intermediate isolates. 
Europe contributed the most isolates over 2009-2012 (54.8\%) (Table 2). $\beta$-lactamase production was reported among $2987(20.2 \%)$ of isolates while $228(1.5 \%)$ isolates were BLNAR (Table 3). H. influenzae was highly susceptible $(>98 \%)$ to most of the agents on the T.E.S.T. panel; the exception to this was ampicillin, for which susceptibility ranged from $68.7 \%$ in Asia/Pacific Rim to $89.0 \%$ in Africa. Globally, there was very little change in $H$. influenzae antimicrobial susceptibility between 2004-2008 and 2009-2012 (Additional file 1: Table S2). The most common isolate sources were respiratory $(71.1 \%)$ and head, ears, eyes, nose and throat (18.4\%) (Table 2).

The global prevalence of $\beta$-lactamase-positive $H$. influenzae decreased significantly $(\mathrm{p}<0.001)$ during this study, from $21.2 \%$ (95\% CI 20.4-22.1) in 2004-2008 to $18.8 \%$ (95\% CI 17.8-19.8) in 2009-2012 (Table 3). The prevalence of $\beta$-lactamase-positive $H$. influenzae was highest in Asia/Pacific Rim (27.8\%) and North America (25.5\%) (Table 3). With the exception of ampicillin (to which $\beta$-lactamase-positive isolates are resistant), susceptibility among $\beta$-lactamase-positive isolates was stable between 2004-2008 and 2009-2012 (Additional file 1: Table S2).

BLNAR isolates were collected from all regions globally and were least common in Middle East (1.0\%) and North America (1.1\%); prevalence was highest in Asia/ Pacific Rim (3.5\%). The global incidence of BLNAR remained consistent during this study, at $1.5 \%$ in 2004 2008 and $1.6 \%$ in 2009-2012 (Table 3). Antimicrobial susceptibility also did not change over the study period (Additional file 1: Table S2).

\section{Discussion}

This report shows that S. pneumoniae global susceptibility has remained relatively stable to most antimicrobial agents globally during the period 2004 to 2012. This reflects the findings of the European Antimicrobial Resistance Surveillance Network (EARS-Net), who showed that non-susceptibility among invasive $S$. pneumoniae in Europe has remained stable between 2004 and 2011, although large variations existed between countries [1]. Results from the SENTRY study in the USA between 1998 and 2011 showed increased resistance among S. pneumoniae to amoxicillin-clavulanate, penicillin (at $\mathrm{MIC} \geq 4 \mathrm{mg} / \mathrm{L}$ ) and ceftriaxone $(18.9 \%, 14.8 \%$ and $11.7 \%$, respectively), although levofloxacin, linezolid, tigecycline and vancomycin retained $100 \%$ activity [16]. Differences between this study and that of Jones et al. [16] may be attributed to the different time period of isolate collection (SENTRY commenced in 1998 compared with the start of TEST in 2004) as well as the source of isolate collection. Also, geography may influence the findings as the current study is a global collection of isolates compared with the USA analysis of Jones et al. [16]. The change in the CLSI breakpoints for S. pneumoniae and penicillin in 2008 also mean that caution must be exercised when comparing susceptibility data between studies [17]. The Jones et al. [16] study used an MIC of $\geq 4 \mathrm{mg} / \mathrm{L}$ for penicillin resistance whereas in this T.E.S.T. study we have used the CLSI penicillin oral breakpoints where resistance is defined as an MIC of $\geq 2 \mathrm{mg} / \mathrm{L}$.

Susceptibility to ceftriaxone and minocycline among $S$. pneumoniae isolates decreased in all the regions; although in the case of ceftriaxone rates of susceptibility remained high. Reasons for these decreases are unknown and warrant further investigation. One factor that may influence susceptibility among S. pneumoniae is the use of the seven- and 13-valent pneumococcal conjugate vaccines (PCV7 and PCV13) which have been shown to modify the epidemiology of pneumococcal disease [18]. The S. pneumoniae collected as part of T.E.S.T. which have elevated ceftriaxone and minocycline MICs warrant molecular analysis to investigate the influence of these vaccines on the resistance profile of $S$. pneumoniae globally.

The high levels of susceptibility to tigecycline among $S$. pneumoniae and $H$. influenzae reported in the current study mirror recent results presented elsewhere. The Assessing Worldwide Antimicrobial Resistance Evaluation Program (AWARE) reported 100\% tigecycline susceptibility among Latin American S. pneumoniae isolates from respiratory tract or complicated skin and soft tissue infections [19]; Zhao et al. reported no tigecycline resistance among 5608 Gram-positive isolates collected in China between 2005 and 2010 [20]; Jones et al. observed 100\% tigecycline susceptibility among $S$ pneumoniae isolates in the USA between 1998 and 2011 [16]; and Nilsson et al. observed good tigecycline activity against $S$. pneumoniae isolates from Northern European countries with a $\mathrm{MIC}_{90}$ of $0.125 \mathrm{mg} / \mathrm{L}$ [21]. Nilsson et al. also reported a low $\mathrm{MIC}_{90}$ $(0.25 \mathrm{mg} / \mathrm{L})$ for tigecycline against $H$. influenzae.

The tigecycline susceptibility results presented here for $S$. pneumoniae are different from those presented by Darabi et al. [12] in their discussion of $S$. pneumoniae collected globally between 2004 and 2008 as a part of the T.E.S.T. study. In the earlier report, S. pneumoniae susceptibility to tigecycline ranged from $87.5 \%$ in Latin America to $97.1 \%$ in the Middle East; in the current report, tigecycline susceptibility was $\geq 99.8 \%$ in all global regions. These changes are likely due to the MIC retesting of several hundred reportedly tigecycline nonsusceptible pneumococci since the publication of Darabi et al. [12]. Re-testing occurred because of the determination that lysed sheep blood in the testing media results in artificially high tigecycline MICs. The re-testing program is carried out by the central laboratory, IHMA, who use un-lysed sheep blood to retest all S. pneumoniae. This program began with the retesting of the most 
recent isolates and then testing backwards to the first isolates received and therefore, some early $S$. pneumoniae MIC results may have been published before all retests were completed. To date, all S. pneumoniae with a tigecycline MIC above $0.06 \mathrm{mg} / \mathrm{L}$ have retested at or below $0.06 \mathrm{mg} / \mathrm{L}$ (data not shown). The difference in results may also be due to the deletion of unevaluable isolates from the T.E.S.T. database. During the production of the dataset used in this manuscript, 236 quarantined S. pneumoniae isolates could not be re-evaluated for susceptibility (i.e., isolates which died on transport from collecting centre to the central laboratory [IHMA], were never shipped to IHMA, or could not be resuscitated for retesting) and were thus permanently removed from the T.E.S.T. database (S. Bouchillon, pers com).

As discussed above, in the current study, oral breakpoints (susceptible $\leq 0.06 \mathrm{mg} / \mathrm{L}$, resistant $\geq 2 \mathrm{mg} / \mathrm{L}$ ) have been used for penicillin against $S$. pneumoniae and not the parenteral breakpoints (meningitis: susceptible $\leq 0.06 \mathrm{mg} / \mathrm{L}$, resistant $\geq 0.12 \mathrm{mg} / \mathrm{L}$; non-meningitis: susceptible $\leq 2 \mathrm{mg} / \mathrm{L}$, resistant $\geq 8 \mathrm{mg} / \mathrm{L}$ ). Use of these oral breakpoints in the current study suggests that penicillin resistance is high globally (PISP, 23.4\%; PRSP, 14.8\%), thus oral penicillin treatment of pneumonia caused by $S$. pneumoniae would appear to be ill-advised. However, the occurrence of $S$. pneumoniae isolates with $\mathrm{MICs} \geq 4 \mathrm{mg} / \mathrm{L}$ is rare worldwide [22], and the use of high-dose parenteral penicillin is generally effective in the treatment of pneumonia caused by drug-resistant S. pneumoniae [23].

$H$. influenzae isolates were highly susceptible to most agents on the T.E.S.T. panel, with global susceptibility ranging from $98.8 \%$ (tigecycline) to $99.9 \%$ (ceftriaxone, levofloxacin, imipenem and meropenem) during the 2004-2012 interval; the sole exception was ampicillin, to which only $78.3 \%$ of isolates were susceptible globally. Regionally, susceptibility in Europe (83.6\%) was higher than in North America (73.4\%). These regional results compare well with Jones et al., who reported $85.2 \%$ and 71.7\% ampicillin susceptibility among $H$. influenzae isolates from Europe and the USA, respectively [24]. Global BLNAR levels were low (1.5\%) in the current study. Japan is known to have a relatively high prevalence of BLNAR H. influenzae [25]; however, no Japanese sites contributed isolates to this study. The USA and Europe, where the majority of T.E.S.T. isolates were collected have lower rates but rates have been reported to be increasing in Europe [26,27]. Studies contemporary with this T.E.S.T. study are scarce making direct comparisons of percentages difficult; however, continued monitoring of these isolates is important, particularly given reports of increasing prevalence.

In the first three years of the T.E.S.T. study, contributing centres in North America were two- to three-fold more numerous than centres from Europe. Since 2008, however, European centres have outnumbered those in North America. This is reflected in the numbers of isolates contributed from these two regions: between 2004 and 2008, 48.3\% of S. pneumoniae and $44.9 \%$ of $H$. influenzae isolates were contributed by centres in North America compared to $31.4 \%$ and $34.5 \%$, respectively, by centres in Europe. When the total study period (20042012) is considered, S. pneumoniae is contributed equally from both regions (40.4\% from North America compared to $40.0 \%$ from Europe); $H$. influenzae isolates from Europe (42.8\%), however, outnumbered those from North America (37.9\%). This shift in prevalence from North America to Europe will undoubtedly have influenced overall susceptibility rates observed over the course of this study, as susceptibility was lower in North America than in Europe for most antimicrobial agents between 2004 and 2012. The number of isolates submitted from the other four regions was similar between the two time periods, contributing approximately 19\% of isolates. The change in the number of North American and European centres over time reinforces the importance of presentation of regional data from global surveillance studies, where possible. This inconsistency in participating centres over time is an inherent weakness of longitudinal surveillance studies, especially international or global surveillance studies. Thus, as is always the case with data derived from multicentre longitudinal surveillance, the trends in antimicrobial susceptibility reported here must be regarded with some caution.

\section{Conclusions}

S. pneumoniae remained highly susceptible to levofloxacin, linezolid, tigecycline and vancomycin while $H$. influenzae was susceptible to most antimicrobial agents in the T.E.S.T. panel (excluding ampicillin). Tigecycline was approved for the treatment of communityacquired bacterial pneumonia in the USA by the FDA in 2009. The results presented in this report show that the in vitro susceptibility of $S$. pneumoniae and $H$. influen$z a e$, two important pathogens which are commonly associated with community-associated pneumonia, has remained high globally in the period since this approval was granted. Tigecycline may thus continue to be an important tool in the armamentarium of physicians for the treatment of CABP.

\section{Additional file}

Additional file 1: Table S1. Antimicrobial susceptibility (MIC ${ }_{90}[\mathrm{mg} / \mathrm{L}]$ and \% susceptibility [\%S]) among S. pneumoniae, PISP and PRSP isolates. Table S2. Antimicrobial susceptibility ( $\mathrm{MIC}_{90}[\mathrm{mg} / \mathrm{L}]$ and \% susceptibility $[\% \mathrm{~S}]$ ) among $H$. influenzae, $\beta$-lactamase-positive $H$. influenzae and BLNAR H. influenzae. 


\section{Competing interests}

VT declares that she has no competing interests. MJD is an employee of Pfizer Inc.

\section{Authors' contributions}

$V T$ was involved in data collection, data interpretation and drafting and reviewing of the manuscript; MJD was involved in study design and participated in data interpretation and the drafting and review of the manuscript. Both authors read and approved the final manuscript.

\section{Acknowledgements}

The authors wish to acknowledge and thank all T.E.S.T. investigators and laboratories for their participation in this study, as well as the staff at IHMA for their coordination of T.E.S.T. This study was sponsored by Pfizer Inc. No authors were paid for their contributions to this manuscript. Medical writing support was provided by Dr. Rod Taylor at Micron Research Ltd, Ely, UK and was funded by Pfizer Inc. Micron Research Ltd also provided data management services which were funded by Pfizer Inc.

\section{Author details}

${ }^{1}$ University Clinic of Respiratory and Allergic Diseases, Golnik 36, 4204 Golnik, Slovenia. ${ }^{2}$ Pfizer Inc, Collegeville, PA, USA.

Received: 25 July 2014 Accepted: 24 October 2014

Published online: 07 November 2014

\section{References}

1. European Centre for Disease Prevention and Control: Antimicrobial resistance surveillance in Europe 2011. Annual Report of the European Antimicrobial Resistance Surveillance Network (EARS-Net). Stockholm: European Centre for Disease Prevention and Control; 2012.

2. Rudan I, O'Brien KL, Nair H, Liu L, Theodoratou E, Qazi S, Lukšić I, Fischer Walker CL, Black RE, Campbell H, Child Health Epidemiology Reference Group (CHERG): Epidemiology and etiology of childhood pneumonia in 2010: estimates of incidence, severe morbidity, mortality, underlying risk factors and causative pathogens for 192 countries. J Glob Health 2013, 3:10401.

3. Mandell LA, Wunderink RG, Anzueto A, Bartlett JG, Campbell GD, Dean NC, Dowell SF, File TM Jr, Musher DM, Niederman MS, Torres A, Whitney CG, Infectious Diseases Society of America; American Thoracic Society: Infectious Diseases Society of America/American Thoracic Society consensus guidelines on the management of community-acquired pneumonia in adults. Clin Infect Dis 2007, 44(Suppl 2):S27-S72.

4. Woodhead M, Blasi F, Ewig S, Garau J, Huchon G, leven M, Ortqvist A, Schaberg T, Torres A, van der Heijden G, Read R, Verheij TJ, Joint Taskforce of the European Respiratory Society and European Society for Clinical Microbiology and Infectious Diseases: Guidelines for the management of adult lower respiratory tract infections-full version. Clin Microbiol Infect 2011, 17(Suppl 6):E1-E59.

5. Lim WS, Baudouin SV, George RC, Hill AT, Jamieson C, Le Jeune I, Macfarlane JT, Read RC, Roberts HJ, Levy ML, Wani M, Woodhead MA, Pneumonia Guidelines Committee of the BTS Standards of Care Committee: BTS guidelines for the management of community acquired pneumonia in adults: update 2009. Thorax 2009, 64(Suppl 3):iii1-iii55.

6. Spindler C, Strålin K, Eriksson L, Hjerdt-Goscinski G, Holmberg H, Lidman C, Nilsson A, Ortqvist A, Hedlund J, Community Acquired Pneumonia Working Group of The Swedish Society of Infectious Diseases: Swedish guidelines on the management of community-acquired pneumonia in immunocompetent adults-Swedish Society of Infectious Diseases 2012. Scand J Infect Dis 2012, 44:885-902.

7. Wiersinga WJ, Bonten MJ, Boersma WG, Jonkers RE, Aleva RM, Kullberg BJ, Schouten JA, Degener JE, Janknegt R, Verheij TJ, Sachs AP, Prins JM, Dutch Working Party on Antibiotic Policy; Dutch Association of Chest Physicians: SWAB/NVALT (Dutch Working Party on Antibiotic Policy and Dutch Association of Chest Physicians) guidelines on the management of community-acquired pneumonia in adults. Neth J Med 2012, 70:90-101.

8. Niederman MS, Luna CM: Community-acquired pneumonia guidelines: a global perspective. Semin Respir Crit Care Med 2012, 33:298-310.

9. Cilli A: The yield of tigecycline in the treatment of community-acquired pneumonia. Tuberk Toraks 2013, 61:155-161.

10. US Food and Drug Administration (FDA): Tigecycline label, 20 March 2009. Rockville, MD: US Food and Drug Administration; 2009. http://www.accessdata. fda.gov/drugsatfda_docs/label/2009/021821s013s017s018lbl.pdf.
11. Tanaseanu C, Bergallo C, Teglia O, Jasovich A, Oliva ME, Dukart G, Dartois N, Cooper CA, Gandjini H, Mallick R, 308 Study Group, 313 Study Group: Integrated results of 2 phase 3 studies comparing tigecycline and levofloxacin in community-acquired pneumonia. Diagn Microbiol Infect Dis 2008, 61:329-338.

12. Darabi A, Hocquet D, Dowzicky MJ: Antimicrobial activity against Streptococcus pneumoniae and Haemophilus influenzae collected globally between 2004 and 2008 as part of the Tigecycline Evaluation and Surveillance Trial. Diagn Microbiol Infect Dis 2010, 67:78-86.

13. Clinical and Laboratory Standards Institute: Performance standards for antimicrobial susceptibility testing: $20^{\text {th }}$ ed. Document M100-S23. Wayne, PA: Clinical and Laboratory Standards Institute; 2013.

14. Clinical and Laboratory Standards Institute: Methods for dilution antimicrobial susceptibility tests for bacteria that grow aerobically, Approved standard: 8th edition. Document M7-A8. Wayne, PA: Clinical and Laboratory Standards Institute; 2009

15. Pfizer Inc. (Wyeth Pharmaceuticals): Tygaci ${ }^{\oplus}$ Product Insert [Internet]. Philadelphia, PA: Pfizer Inc., 2013. http://www.pfizerpro.com/hcp/tygacil.

16. Jones RN, Sader HS, Mendes RE, Flamm RK: Update on antimicrobial susceptibility trends among Streptococcus pneumoniae in the United States: report of ceftaroline activity from the SENTRY Antimicrobial Surveillance Program (1998-2011). Diagn Microbiol Infect Dis 2013, 75:107-109.

17. Weinstein MP, Klugman KP, Jones RN: Rationale for revised penicillin susceptibility breakpoints versus Streptococcus pneumoniae: coping with antimicrobial susceptibility in an era of resistance. Clin Infect Dis 2009, 48:1596-1600.

18. Richter SS, Heilmann KP, Dohrn CL, Riahi F, Diekema DJ, Doern GV: Pneumococcal serotypes before and after introduction of conjugate vaccines, United States, 1999-2011. Emerg Infect Dis 2013, 19:1074-1083.

19. Flamm RK, Sader HS, Jones RN: Spectrum and potency of ceftaroline against leading pathogens causing community-acquired respiratory tract and skin and soft tissue infections in Latin America, 2010. Braz J Infect Dis 2013, 17:564-572.

20. Zhao C, Sun H, Wang H, Liu Y, Hu B, Yu Y, Sun Z, Chu Y, Cao B, Liao K, Lei J, Hu Z, Zhang L, Zhang X, Xu Y, Wang Z, Chen M: Antimicrobial resistance trends among 5608 clinical Gram-positive isolates in China: results from the Gram-Positive Cocci Resistance Surveillance program (2005-2010). Diagn Microbiol Infect Dis 2012, 73:174-181.

21. Nilsson LE, Frimodt-Møller N, Vaara M, Simonsen GS, Tigecycline Study Group: Comparative activity of tigecycline and tetracycline on Gram-negative and Gram-positive bacteria revealed by a multicentre study in four North European countries. Scand J Infect Dis 2011, 43:707-713.

22. Liñares J, Ardanuy C, Pallares R, Fenoll A: Changes in antimicrobial resistance, serotypes and genotypes in Streptococcus pneumoniae over a 30-year period. Clin Microbiol Infect 2010, 16:402-410.

23. Jinno S, Jacobs MR: Pneumonia due to drug-resistant Streptococcus pneumoniae. Curr Infect Dis Rep 2012, 14:292-299.

24. Jones RN, Farrell DJ, Mendes RE, Sader HS: Comparative ceftaroline activity tested against pathogens associated with community-acquired pneumonia: results from an international surveillance study. J Antimicrob Chemother 2011, 66(Suppl 3):iii69-iii80.

25. Yamaguchi K, Ohno A, Ishii Y, Tateda K, Iwata M, Levofloxacin-Surveillance Group: In vitro susceptibilities to levofloxacin and various antibacterial agents of 12,866 clinical isolates obtained from 72 centers in 2010. Jpn J Antibiot 2012, 65:181-206.

26. Fluit AC, Florijn A, Verhoef J, Milatovic D: Susceptibility of European $\beta$-lactamase-positive and -negative Haemophilus influenzae isolates from the periods 1997/1998 and 2002/2003. J Antimicrob Chemother 2005, 56:133-138.

27. Heilmann KP, Rice CL, Miller AL, Miller NJ, Beekmann SE, Pfaller MA, Richter SS, Doern GV: Decreasing prevalence of $\beta$-lactamase production among respiratory tract isolates of Haemophilus influenzae in the United States. Antimicrob Agents Chemother 2005, 49:2561-2564.

doi:10.1186/s12941-014-0052-2

Cite this article as: Tomic and Dowzicky: Regional and global antimicrobial susceptibility among isolates of Streptococcus pneumoniae and Haemophilus influenzae collected as part of the Tigecycline Evaluation and Surveillance Trial (T.E.S.T.) from 2009 to 2012 and comparison with previous years of T.E.S.T. (2004-2008). Annals of Clinical Microbiology and Antimicrobials 2014 13:52. 